\title{
Reemplazos de directivos en México: de la teoría a la práctica
}

Karen Watkins Fassler Universidad Popular Autónoma del Estado de Puebla karen.watkins@upaep.mx

\section{Martín Dávila Delgado} Universidad Popular Autónoma del Estado de Puebla martin.davila@upaep.mx

\section{Resumen}

En este trabajo se reflexiona sobre la teoría de los reemplazos y su aplicación al caso de México. Se buscan las correspondencias, así como las diferencias, entre la literatura para países desarrollados y la realidad mexicana (economía emergente). Se describe no sólo la ocurrencia de los reemplazos en México, sino también sus repercusiones sobre el desempeño empresarial. Se concluye que en México hay muy pocos reemplazos, particularmente por la estructura familiar de las firmas mexicanas; asimismo, éstos no favorecen al desempeño empresarial, al menos en el corto y mediano plazo.

Palabras clave: reemplazos, gobierno corporativo, desempeño empresarial, México. 


\title{
Turnovers in Mexican firms: from theory to practice
}

\begin{abstract}
This paper studies the turnover theory and its application in Mexico. We look for correspondences as well as differences between the literature for developed countries and the Mexican reality (emerging market). We describe both the occurrence of turnovers and their effects on firm performance. We conclude that in Mexico there are very few turnovers, particularly because of the family structure of Mexican enterprises. In addition, these turnovers did not favor firm performance, at least in the short and medium terms.
\end{abstract}

Keywords: turnovers, corporate governance, firm performance, Mexico.

\section{Introducción}

Los reemplazos de ejecutivos y directivos empresariales tienen lugar por diversas razones. La literatura señala que existen motivaciones internas y externas a las firmas, que repercuten en la probabilidad de sustituir a estos funcionarios. Dentro de los elementos internos se encuentran el desempeño empresarial, la renuncia o jubilación del agente en cuestión y el cambio en la propiedad de la empresa, entre otros. Sobre los causantes externos se mencionan, por ejemplo, al mercado laboral y las variaciones del entorno empresarial. Cualquiera que sea la razón para que se dé el reemplazo, lo cierto es que éste tiene repercusiones importantes sobre el destino de la firma y su desempeño.

En este trabajo se reflexiona sobre la teoría de los reemplazos y su aplicación al caso de México. Se buscan las correspondencias, así como las diferencias, entre la literatura para países desarrollados y la realidad mexicana (economía emergente). Existe muy poca información sobre este tema para América Latina, y particularmente para México, lo cual lo hace relevante. Se describe no sólo la ocurrencia de los reemplazos en México, sino también sus repercusiones sobre el desempeño empresarial.

El estudio se organiza de la siguiente manera: en la sección siguiente se aborda la teoría de los reemplazos; más adelante se expone el tema del desempeño empresarial ex ante y ex post al reemplazo; posteriormente, se estudian los reemplazos de directivos en México; finalmente, se presentan las conclusiones y reflexiones finales del estudio. 


\section{Teoría de reemplazos}

Las empresas son organizaciones sociales, lo cual implica que se comportan de forma dinámica, cambiante, evolutiva. El reemplazo tanto de altos ejecutivos como de miembros de las juntas directivas toma lugar en ciertos periodos, de forma sorpresiva o esperada. Estos cambios ocurren tanto por situaciones internas como externas a las firmas. Desde el punto de vista externo, pueden darse reemplazos cuando se presentan variaciones de entorno que lo ameriten. Ejemplo de ello resulta la necesidad de ajustar el perfil del ejecutivo o del director por la evolución del mercado o la industria a la cual pertenece la empresa; una fusión, la apertura del mercado, la internacionalización, la presión de inversionistas institucionales, entre otros factores, pueden ser causas del reemplazo (ver Hermalin y Weisbach, 1998; Fee y Hadlock, 2000; Lefort y Walker, 2000; Fung y Rui, 2006; Jenter y Kanaan, 2006). Otro elemento externo lo constituye la evolución y flexibilidad del mercado laboral de ejecutivos y/o directivos.

Si estos mercados se vuelven más abiertos y competitivos, entonces la probabilidad de reemplazo se incrementa (ver Manne, 1965; Fama, 1980; Fama y Jensen, 1983). Desde el punto de vista interno, un cambio de dueños, la renuncia o jubilación del ejecutivo o directivo, así como el surgimiento de un mercado laboral interno competitivo representan variaciones en la organización que fomentan el reemplazo (ver Nair, 2005). También desde la óptica interna, el desempeño de los funcionarios y de la firma es un factor de decisión importante para el reemplazo. El despido tanto de altos ejecutivos como de miembros de la junta de directores ocurre generalmente por una mala gestión y bajos rendimientos empresariales (ver Jovanovic, 1979; Coughlan y Schmidt, 1985; McKenna, 1986; Murphy, 1986; Warner et al., 1988; Weisbach, 1988; Conyon y Florou, 2002; Fee y Hadlock, 2004). Las empresas que experimentan rendimientos pobres vigilan más de cerca a sus funcionarios, en contraste a las situaciones en las cuales las empresas se encuentran en bonanza.

Los buenos ejecutivos y directivos son difíciles de encontrar y tienen mucha demanda. Encontrar un nuevo funcionario es una tarea crítica, complicada y riesgosa debido a la complejidad involucrada en el proceso, así como el impacto significativo que esta figura puede tener sobre el desempeño de la firma. El reemplazo del agente puede afectar al desempeño empresarial y, a su vez, el desempeño empresarial puede afectar la probabilidad del reemplazo. Algunas veces el reemplazo será benéfico para la firma, pero en ocasiones será dañino. Cuando la firma tiene 
un desempeño pobre, se busca que el reemplazo resucite a la firma; mientras que cuando la empresa es exitosa y se da un reemplazo, se busca a alguien con las cualidades y características necesarias que mantengan ese éxito.

\section{Desempeño empresarial ex ante al reemplazo}

La mayoría de los reemplazos ocurre cuando el desempeño empresarial ha sido pobre. Dentro del Gobierno Corporativo, uno de los mecanismos que se utiliza para disciplinar a los administradores y directores es la sustitución de los mismos. Autores como Coughlan y Schmidt (1985); Warner et al. (1988); Weisbach (1988); Furtado y Karan (1990); Kim (1996); y Lausten (2002) encuentran una asociación negativa entre el desempeño corporativo y los cambios de altos funcionarios. Barro y Barro (1990); Jensen y Murphy (1990); Gibbons y Murphy (1990); Kaplan (1994); Denis y Denis (1995); Cosh y Hughes (1997); Parrino (1997); Coffee (1999); DeFond y Park (1999); y Huson et al. (2004) presentan evidencia de que los funcionarios son forzados a dejar las empresas debido a una larga y significativa caída en el desempeño de las firmas.

Furtado y Karan (1990) y Khanna y Poulsen (1995) mencionan que algunos de los reemplazos que se dan después de un pobre desempeño empresarial se deben a lo que llaman el "chivo expiatorio". Esto es, se culpa a los administradores y directivos de la disminución del desempeño empresarial, aunque ellos no sean los responsables directos. Puede que los bajos rendimientos se deban a una recesión en el mercado, a competencia desleal por parte de otras empresas, a elementos macroeconómicos e internacionales externos a la empresa; en suma, factores que se escapan del control de los funcionarios.

La diferencia entre el desempeño actual de la firma y las expectativas sobre el mismo que tienen los accionistas puede representar adicionalmente un indicador para la posibilidad de reemplazo (ver Puffer y Weintrop, 1991). Los cambios en la alta dirección ocurren muchas veces cuando el desempeño es menor, en comparación con las expectativas que se tienen sobre dicho desempeño.

\section{Desempeño empresarial ex post al reemplazo}

Los inversionistas generalmente interpretan de forma favorable los anuncios de reemplazos de los administradores y directivos, debido a que ellos esperan que éstos provoquen en promedio un mejoramiento en el desempeño financiero de la 
firma. Estas expectativas, al menos en el corto plazo, pueden generar ascensos en el rendimiento empresarial (particularmente a través del incremento del valor de mercado de la empresa; ver Weisbach (1988); Bonnier y Bruner (1989); Denis y Denis (1995); y Hotchkiss (1995)).

Huson et al. (2004) examinan la relación entre el reemplazo de los altos ejecutivos y el posterior desempeño financiero de las firmas. Los indicadores contables de desempeño muestran una mejora luego del reemplazo. El grado de progreso está directamente relacionado con el número de accionistas institucionales, la presencia de miembros externos que dominen la junta de directores, así como el nombramiento en el puesto de director general (CEO) a un agente externo ajeno a la empresa.

Brunello et al. (2003) estudian la relación entre el desempeño de la firma y cambios en el CEO y otros ejecutivos, así como miembros de la junta de directores, para empresas listadas en el mercado accionario de Italia de 1988 a 1996. Para ello, contemplan tres variables de reemplazo: 1) cambio de varios miembros de la junta directiva, por ejemplo, cambio del presidente, del vicepresidente o de todos los miembros de la junta de directores; 2) cambio del presidente de la junta directiva y del CEO; 3) cambio únicamente del CEO. Se obtienen diferentes resultados para los tres indicadores de reemplazo. La más notable conclusión a la que llegan es la referente a la carencia de correlación entre el cambio solamente del CEO y cualquier variable de desempeño de la firma, lo que hace pensar que el reemplazo del CEO no afecta al desempeño empresarial per se.

Por el contrario, Khanna y Poulsen (1995) encuentran evidencia empírica que una vez que se da el reemplazo del CEO de la compañía, el desempeño de la empresa tiende a caer. Esto ocurre cuando el nuevo CEO no es el más apto para dirigir la organización; esto es, sus habilidades y las estrategias emprendidas por éste no son las adecuadas. Otro de los argumentos empleados para explicar esta situación se refiere a la falta de experiencia y conocimiento interno del nuevo ejecutivo.

\section{Reemplazos de directivos en México}

Para analizar la presencia de los reemplazos de directivos en México, así como sus repercusiones sobre el desempeño empresarial, se recopiló información sobre 176 firmas no financieras que cotizaron en la Bolsa Mexicana de Valores (BMV) de 1990 a 2009. Este horizonte temporal permite estudiar los reemplazos en épocas 
de crisis empresarial (1994-1995 y 2008-2009), así como en situaciones normales previas y posteriores a las mismas. Asimismo, a raíz de la crisis mexicana de 1994-1995, se dieron en México cambios importantes en la regulación corporativa; particularmente, se estableció la Ley del Mercado de Valores $(2001,2007)$ y el Código de Buenas Prácticas Corporativas $(1999,2006)$. Estas normas repercutieron en la gobernanza de las empresas y consecuentemente en la probabilidad de reemplazos de directivos.

Para los primeros años del periodo de nuestra investigación, los datos financieros se obtienen directamente de la Bolsa Mexicana de Valores. ${ }^{1}$ Esta información se encuentra disponible a manera de microfilmación. La gran mayoría de los datos es obtenida de manera electrónica en el Sistema Integral de Valores Automatizados (SIVA, por sus siglas en español), así como en Infosel Financiero. La información sobre los miembros de las juntas directivas se registra de manera manual del Anuario Financiero que se encuentra en el Centro de Información ${ }^{2}$ de la BMV.

Se toma como variable de reemplazo al cambio de los presidentes de los consejos de administración (juntas directivas) en México. ${ }^{3}$ Se entiende por reemplazo el cambio del presidente de la junta directiva de una firma en un año en particular. Cabe mencionar que de 1990 a 2009 una misma empresa puede presentar más de un reemplazo.

Como variable de desempeño empresarial se utiliza el rendimiento sobre los activos (ROA). Éste se calcula como la utilidad antes de intereses e impuestos entre los activos totales. Para cada empresa, el ROA previo al reemplazo del presidente de la junta directiva se refiere al observado el año anterior al mismo; el ROA durante el reemplazo es aquel propiamente en el año en que se da este evento; el ROA posterior al reemplazo se refiere al observado un año luego del cambio de este funcionario. Para calcular el ROA promedio previo, durante y posterior al reemplazo de los presidentes de las juntas directivas de las empresas en cuestión, se hace uso de un promedio simple por periodo.

\footnotetext{
${ }^{1}$ Se agradece a Gonzalo Castañeda por permitir el acceso a su base de datos financieros de las empresas, a Jaime Díaz por proporcionar el acceso al SIVA.

${ }^{2}$ Se agradece a Simón Cruz por la ayuda en la recopilación de datos en el Centro de Información de la BMV; asimismo, se agradece a Ana Antón, Montserrat Ángel, Érick Sánchez y Manuel Garzón por su colaboración en la captura de los nombres de los miembros de las juntas directivas. La base de datos fue elaborada por el doctor Martín Dávila; cualquier error es responsabilidad del autor.

${ }^{3}$ En México, la información histórica de los altos ejecutivos no es pública; por ende, se trabaja exclusivamente con los presidentes de los consejos de administración.
} 
De acuerdo con la información obtenida de toda la muestra, en total se presentaron reemplazos en 654 ocasiones. Dado que cada cambio de año representa un posible reemplazo y son 176 las empresas contempladas, el mayor número factible de reemplazos es de 3,344. Esto implica que los reemplazos ocurrieron sólo en el 19.6\% de los casos posibles. Para el primer periodo de precrisis (1990-1993) únicamente se tuvieron 48 reemplazos, los que empezaron a aumentar a través del tiempo, con una sola ruptura en el año 2001. Este año coincide con la reforma de la Ley del Mercado de Valores, donde se incorpora que el 25\% de los consejeros deben ser independientes de acuerdo con las buenas prácticas de gobierno corporativo. La posibilidad de sustituir agentes, tales como el director general o el presidente del consejo de administración de una compañía, favorece la alineación de objetivos empresariales. La Porta et al. (1998); Claessens et al. (2002); y Dyck y Zingales (2004) sostienen que el sistema legal protege a los inversionistas mediante el otorgamiento de derechos para disciplinar a los agentes por medio de su reemplazo. Lo anterior puede ser visto como una forma para hacer cumplir los contratos tal y como están diseñados, de manera que limiten a los agentes de los beneficios privados obtenidos mediante el control de las firmas. En este sentido, los reemplazos constituyen un mecanismo de gobierno corporativo (ver también Manne, 1965; Harrison et al., 1988; y Gibson, 2003).

Por otro lado, existen otras medidas de gobierno corporativo que a su vez se relacionan con los reemplazos. El incorporar una mayor proporción de miembros externos a las empresas en las juntas directivas constituye un mecanismo más de gobierno corporativo, con lo cual puede disminuir la práctica de los reemplazos. De acuerdo con la literatura, los directores externos disciplinan más a los ejecutivos, por los que los flujos de efectivo son mejor administrados que en firmas con una baja proporción de consejeros independientes. Lo anterior lo corroboran autores tales como Brickley et al. (1994); Dehaene et al. (2001); Hossain et al. (2001); así como Lefort y González (2010).

Para el primer periodo de crisis (1994-1995) se dieron 80 reemplazos y en la poscrisis (1996-2007) se llegó a 443 reemplazos. Para los años 2008 y 2009, que coinciden con la actual crisis financiera mundial, se registraron 83 reemplazos. En la siguiente gráfica se muestran el número de reemplazos por cada año contemplado. 
Gráfica 1

Número de reemplazos de los presidentes de los

Consejos de Administración en México, de 1990 a 2009

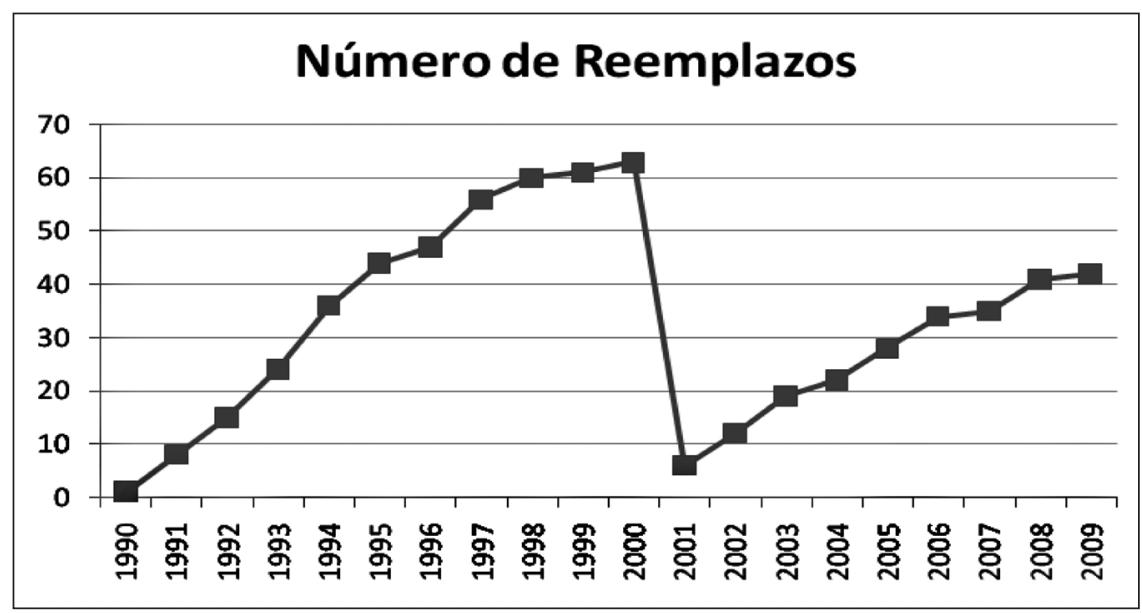

Si bien es cierto que el rendimiento empresarial en promedio disminuye durante los periodos de crisis (ver gráfica siguiente), no se observa un mayor número de reemplazos durante estos episodios. En promedio se registran 40 reemplazos anuales durante la crisis de 1994-1995, consistente con la crisis reciente (20082009), donde este número es de 41.5.

En comparación con los años 1996-2007 (poscrisis), no se aprecian diferencias significativas, ya que en promedio el número de reemplazos en este periodo es de 36.9 por año. La realidad mexicana muestra más bien una tendencia creciente en el número de reemplazos a través del tiempo, con excepción del quiebre durante 2001. Esto proporciona evidencia en contra de la hipótesis del "chivo expiatorio" de Furtado y Karan (1990), y Khanna y Poulsen (1995). 


\section{Gráfica 2}

ROA promedio durante los periodos de precrisis (1990-1993), crisis (1994-1995), poscrisis (1996-2007) y la crisis reciente (2008-2009) en México

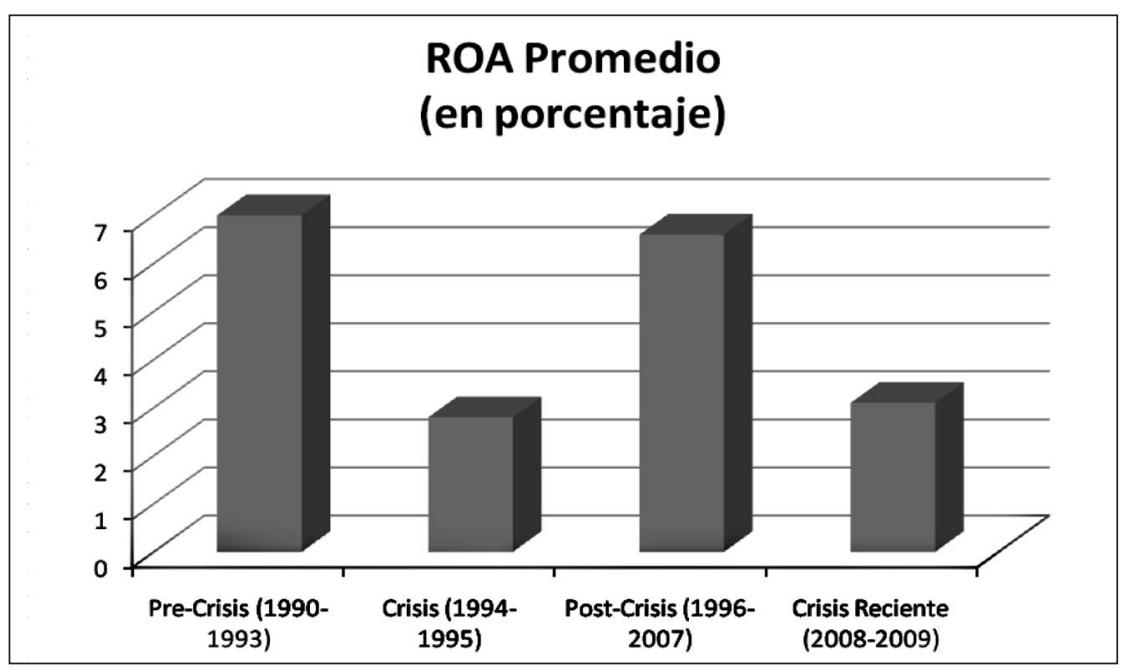

Una explicación tentativa para los relativamente pocos reemplazos observados en las empresas mexicanas es la alta incidencia de dueños en las juntas directivas de estas firmas (aproximadamente el 70\% de las empresas en México son familiares; ver Dávila y Watkins, 2008).

En este tipo de compañías, los determinantes de los reemplazos estudiados en la literatura para países desarrollados pierden validez, pues difícilmente se sustituye a un ejecutivo o directivo que pertenece a la familia empresarial. De hecho, se observa gran estabilidad en estos puestos, lo cual favorece el argumento anterior; 112 de las 176 empresas de la muestra (64\% de las mismas) no presentaron reemplazos durante el periodo de estudio 1990-2009. Particularmente, para las empresas familiares, se observaron reemplazos en 475 ocasiones, $20 \%$ del total de 2,341 observaciones.

Con respecto a la relación entre el desempeño empresarial y los reemplazos, se aprecia que en el $25 \%$ de los casos, el rendimiento previo al reemplazo fue negativo. Esto indica que el cambio en el presidente de la junta directiva pudo ocasionarse efectivamente como un castigo por mal desempeño. Cabe mencionar que la 
gran mayoría de las veces (73\%) estos reemplazos se dan dentro de las mismas familias que controlan a una firma, por lo que no se afecta la estructura familiar de la empresa. En el restante $27 \%$ de los casos el reemplazo se debió a una recomposición en el control de la empresa, donde la familia que controlaba a la firma deja de ser su dueña.

En general, el reemplazo del presidente de la junta directiva no favoreció al desempeño empresarial ni en el corto plazo (durante el año del reemplazo) ni en el mediano plazo (un año posterior al cambio). Algunas excepciones notables de esto se dieron en aquellas empresas con desempeño muy pobre antes del reemplazo. En el año previo al reemplazo, el ROA promedio fue de $4.5 \%$. Durante el año propiamente del reemplazo, éste se mantuvo prácticamente igual, en $4.7 \%$. Posterior a este evento, el ROA promedio creció ligeramente ubicándose en 5\%.

\section{Gráfica 3}

ROA promedio antes, durante y posterior al reemplazo

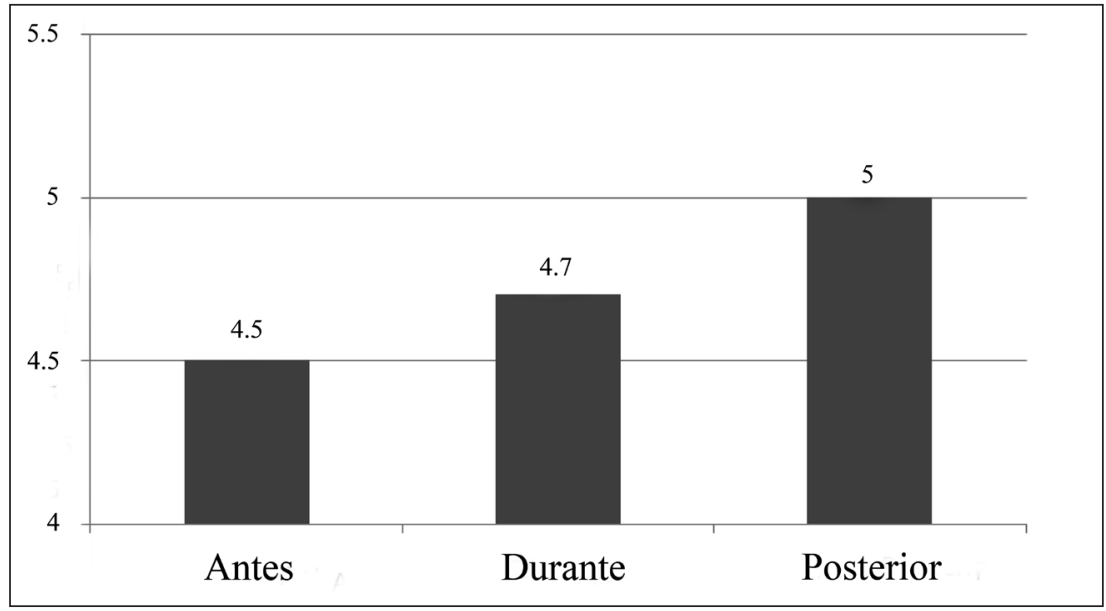

\section{Conclusiones y reflexiones finales}

Los resultados de este trabajo muestran que la realidad mexicana no concuerda plenamente con la literatura revisada sobre el tema para países desarrollados. En particular, en los veinte años contemplados pocas veces se dan los reemplazos, aun en presencia de factores determinantes tales como un pobre desempeño empresarial, cambios en el entorno de las firmas y mayor competencia en el mercado 
laboral. Una explicación probable para ello es el hecho de que la gran mayoría de las firmas en México es familiar, por lo que sus directivos no son reemplazados por pertenecer a la familia empresarial. Ésta es una realidad en muchos países latinoamericanos, donde no hay separación entre propiedad y control empresarial (ver Lefort y Walker, 2000; Santiago y Brown, 2009).

La falta de reemplazos tiene repercusiones importantes sobre el destino de las compañías. De acuerdo con la teoría de agencias, si un funcionario no desempeña debidamente su trabajo, éste es sustituido por otro con lo que se garantiza en cierta medida la sobrevivencia de la firma a través del tiempo. La amenaza creíble del reemplazo permite que se dé la alineación de incentivos entre el agente y el principal —el dueño- quien busca maximizar el valor de las acciones y por ende las utilidades de la empresa. Si bien existen otras maneras de alinear los objetivos, el reemplazo es sin lugar a dudas uno de los mecanismos más eficientes para este fin. En el caso de las empresas mexicanas, la teoría de agencias pierde importancia debido a que el agente y el principal son la misma figura, pertenecen a la misma familia. ${ }^{4}$ No existe un conflicto de intereses debido a que no hay una separación entre la propiedad y el control de la firma. No obstante, esto no garantiza que se maximice el valor de la empresa, ya que no necesariamente los miembros de la familia son los mejores empresarios o directivos. El gobierno corporativo debe redefinirse entonces para contemplar la empresa familiar. Es de vital importancia establecer medidas de gobierno corporativo propias para la empresa familiar, que favorezcan sus resultados y su continuidad.

La realidad muestra que las empresas familiares no llegan a sobrevivir la cuarta generación. De acuerdo con estudios pasados (Morris, 1997), únicamente el 30\% de estas empresas logran sobrepasar la segunda generación familiar. Este porcentaje cae a $15 \%$ para la tercera generación. Las razones para lo anterior son muchas, sin embargo la principal se refiere a problemas de sucesión (reemplazos) por falta de planeación. El éxito de la empresa familiar depende de la figura del fundador, aquel miembro de la familia que tuvo la visión y llevó las riendas de la corporación; cuando esta persona hereda la compañía surgen los conflictos, pues el sucesor generalmente no tiene las mismas competencias, visión ni credibilidad. Por razones humanas y de costos, el fundador suele darle poca importancia al establecimiento de un plan de sucesión completo, el cual incorpora no sólo la planeación misma

${ }^{4}$ Para una descripción detallada de la gobernanza empresarial en México ver Husted y Serrano, 2001. 
sino también consideraciones sobre el proceso de sucesión y la influencia familiar. Desde el punto de vista humano, ¿quién piensa en dejar de trabajar o morir? Por otro lado, los planes de sucesión son sumamente costosos; en especial para firmas pequeñas, la realización de los mismos se vuelve inalcanzable.

Dada la importancia de las empresas familiares en México y la problemática de su permanencia en el tiempo, resulta fundamental realizar una investigación futura que contemple los siguientes puntos: primero, que ofrezca información pertinente sobre la organización de la empresa familiar y sus finanzas. Hay poca información sobre las empresas familiares, pues la mayoría de éstas son pequeñas y, por ende, no están listadas en bolsa (es decir, su información no es de acceso público); segundo, indagar sobre la existencia de elementos que repercutan en la permanencia de la empresa en el tiempo, particularmente lo que se refiere a planes de sucesión y composición y existencia de órganos internos tales como la junta directiva. Algunos autores tales como Santiago y Brown (2009) y Lefort y González (2010) ya han analizado los beneficios de los consejos de administración independientes sobre la gobernanza de las empresas familiares latinoamericanas. Finalmente, es importante establecer políticas de buen gobierno corporativo propias para este tipo de organizaciones.

\section{Referencias}

Barro, J. y R. Barro (1990). Pay, performance and turnover of Bank CEOs. Journal of Labor Economics (8): 448-481.

Bonnier, K. A. y R.F. Bruner (1989). An analysis of stock price reaction to management change in distressed firms. Journal of Accounting and Economics (11): 95-106.

Brickley, J.A., J. Coles y R.L. Terry (1994). Outside directors and the adoption of poison pills. Journal of Financial Economics (35): 371-390.

Brunello, Giorgio, Clara Graziano y Bruno Parigi (2003). CEO Turnover in insider dominated boards: the italian case. Journal of Banking and Finance (27): 1027-1051. 
Claessens, Stijn, Simeon Djankov, Joseph P.H. Fan y H.P. Lang Larry (2002). Disentangling the incentive and entrenchment effects of large shareholdings. Journal of Finance (57): 2741-2772.

Coffee, J. (1999). The future as history: the prospects for global convergence in corporate governance and its implications. Northwestern University Law Review (93): 641-708.

Conyon, Martin J. y Annita Florou (2002). Top executive dismissal, ownership and corporate performance. Accounting and Business Research (32): 209-226.

Coughlan, Anne T. y Ronald M. Schmidt (1985). Executive compensation, management turnover and firm performance: an empirical investigation. Journal of Accounting and Economics (7): 43-66.

Cosh, A. y A. Hughes (1997). Executive remuneration, executive dismissal and institutional shareholdings. International Journal of Industrial Organization (15): 469-492.

Dávila y Watkins (2008). Corporate governance and firm performance lessons from de Mexican crisis (1994-1995). Making Capital Markets Work Through Corporate Governance, ed. World Council for Corporate Governance

DeFond, Mark L. y C.W. Park (1999). The effect of competition en CEO turnover. Journal of Accounting and Economics (27): 35-56.

Dehaene, Alexander, Veerle De Vuyst y Ooghe Hubert (2001). Corporate performance and board structure in Belgian companies. Long Range Planning (34): 383-398.

Denis, D.J. y Diane K. Denis (1995). Performance changes following top management dismissals. Journal of Finance (50): 1029-1057.

Dyck, Alexander y Luigi Zingales (2004). Private benefits of control: an international comparison. Journal of Finance (59): 537-600.

Fama, Eugene F. (1980). Agency problems and the theory of the firm. Journal of Political Economy (88): 288-307. 
y C. Jensen Michael (1983). Separation of ownership and control. Journal of Law and Economics (26): 301-325.

Fee, C. Edward y Charles J. Hadlock (2000). Management turnover and product market competition: empirical evidence from the U.S. newspaper industry. Journal of Business (73): 205-243.

(2004). Management turnover across the corporate hierarchy, Journal of Accounting and Economics (37): 3-38.

Fung, Peter M.Y. y Oliver M. Rui (2006). Firm performance, governance structure, and top management turnover in a transitional economy. Journal of Management Studies (43): 1289-1330.

Furtado, E.P.H. y V. Karan (1990). Causes, consequences and shareholder wealth effects of management turnover: a review of the empirical evidence. Financial Management (19): 60-75.

Gibbons, R. y K.J. Murphy (1990). Relative performance evaluation for Chief Executive Officers. Industrial and Labor Relations Review (43): 305-515.

Gibson, Michael S. (2003). Is corporate governance ineffective in emerging markets? Journal of Financial and Quantitative Analysis (38): 231-250.

Harrison, J.R., D.L. Torres y S. Kukalis (1988). The changing of the guard: turnover and structural change in the top-management positions. Administrative Science Quarterly (33): 211-232.

Hermalin, Benjamin E. y S. Weisbach Michael (1998). Endogenously chosen boards of directors and their monitoring of the CEO, The American Economic Review (88): 96-118.

Hossain, Mahmud, Andrew K. Prevost y Ramesh P. Rao (2001). Corporate governance in New Zealand: the effect of the 1993 companies act on the relation between board composition and firm performance. Pacific-Basin Finance Journal (9): 119-145. 
Hotchkiss, Edith S. (1995). Postbankruptcy performance and management turnover, Journal of Finance (50): 3-21.

Huson, Mark R., Paul H. Malatesta y Robert Parrino (2004). Managerial succession and firm performance. Journal of Financial Economics (74): 237-275.

Husted, Bryan W. y Carlos Serrano (2001). Corporate governance in Mexico, Instituto Tecnológico y de Estudios Superiores de Monterrey, documento de trabajo, 1-28.

Jensen, Michael C. y K.J. Murphy (1990). Performance pay and top management incentives. Journal of Political Economy (98): 225-264.

Jenter, Dirk C. y Fadi Kanaan (2006). CEO turnover and relative performance evaluation. MIT Sloan Research Paper (4594-06).

Jovanovic, Boyan (1979). Job matching and the theory of turnover. Journal of Political Economy (87): 972-990.

Khanna, Naveen y Annette B. Poulsen (1995). Managers of financially distressed firms: villains or scapegoats? Journal of Finance (50): 919-940.

Kaplan, Steven N. (1994). Top executives, turnover, and firm performance in Germany. Journal of Law Economics and Organization (10): 142-159.

Kim, Y. (1996). Long-term firm performance and chief executive turnover: an empirical study of the dynamics. Journal of Law, Economics, and Organization (12): 480-496.

La Porta, Rafael, Florencio López-de-Silanes, Andrei Shleifer y Robert W. Vishny (1998). Law and finance. Journal of Political Economy (106): 1115-1155.

Lausten, Mette (2002). CEO turnover, firm performance and corporate governance: empirical evidence on Danish firms. International Journal of Industrial Organization (20): 391-414.

Lefort, Fernando y Eduardo Walker (2000). Corporate governance: challenges for Latin America. Revista ABANTE (2): 99-111. 
Lefort, Fernando y Rodrigo González (2010). Hacia un mejor gobierno corporativo en Chile. Revista ABANTE 11 (en prensa).

Manne, Henry G. (1965). Mergers and the market for corporate control. Journal of Political Economy 73 (2): 110-120.

McKenna, C. J. (1986). Equilibrium wage offers and turnover in a simple search market. The Economic Journal (96): 785-797.

Morris, Michael (1997). Correlates of success in family business transitions. Journal of Business Venturing (12): 385-401.

Murphy, Kevin J. (1986). Incentives, learning and compensation: a theoretical and empirical investigation of managerial labor contracts. Rand Journal of Economics (17): 59-76.

Nair, Vinay B. (2005). Corporate governance and internal organization, Wharton School at University of Pennsylvania, working paper.

Parrino, Robert (1997). CEO turnover and outside succession: a cross-sectional analysis. Journal of Financial Economics (46): 165-197.

Puffer, S.M. y J.B. Weintrop (1991). Corporate performance and CEO turnover: the role of performance expectations. Administrative Science Quarterly (36): 1-19.

Santiago, Marisela y Cynthia Brown (2009). An empirical analysis of Latin American board of directors and minority shareholders' rights. Forum Empresarial (14): 1-18.

Warner, Jerold B., Ross L. Watts y Karen H. Wruck, (1988). Stock prices and top management changes. Journal of Financial Economics (20): 461-492.

Weisbach, Michael S. (1988). Outside directors and CEO Turnover. Journal of Financial Economics (20): 431-460. 